\title{
Resolution Uniformity and Sensitivity of the NIH ATLAS Small Animal PET Scanner: Comparison to Simulated LSO Scanners Without Depth-of-Interaction Capability
}

\author{
Jürgen Seidel, Juan José Vaquero, Senior Member, IEEE, and Michael V. Green
}

\begin{abstract}
PET scanners designed to image animals the size of rats and mice should possess simultaneously high and uniform spatial resolution and high sensitivity. ATLAS (Advanced Technology Laboratory Animal Scanner), an $11.8 \mathrm{~cm}$ diameter aperture, $2 \mathrm{~cm}$ axial field-of-view ring-type research scanner seeks these goals by surrounding the animal with eighteen 15 mm deep, LGSO (7 mm) / GSO (8 mm) phoswich detector modules.
\end{abstract}

A Monte Carlo simulation was used to compare the variation of resolution across the field-of-view and the absolute central point source sensitivity (ACS) of ATLAS to similar systems comprised only of LSO arrays of different depths with no depth-of-interaction (DOI) capability. For ATLAS radial spatial resolution deteriorated by $27 \%$ from the center to $3 \mathrm{~cm}$ off-axis. Scanners comprised of $15 \mathrm{~mm}$ deep, 10 $\mathrm{mm}$ deep and $7 \mathrm{~mm}$ deep LSO crystals deteriorated by $100 \%$, $51 \%$, and $20 \%$ respectively, over the same distance. Simulated ACS (absorbed energies $\geq 250 \mathrm{keV}$ ) for ATLAS was $2.0 \%$ and for the $15 \mathrm{~mm}, 10 \mathrm{~mm}$ deep and $7 \mathrm{~mm}$ deep LSO scanners $2.4 \%$, $1.5 \%$, and $0.9 \%$, respectively.

Radial resolution loss $3 \mathrm{~cm}$ off-axis and ACS measured for the actual ATLAS scanner were similar to the values obtained by simulation ( $27 \%$ resolution loss, $1.8 \%$ ACS). The phoswich design thus achieves good resolution uniformity over a $6 \mathrm{~cm}$ FOV while preserving sensitivity compared to equivalent nonDOI LSO scanners with a range of crystal depths.

\section{INTRODUCTION}

A small animal PET scanner should have high sensitivity and high and uniform spatial resolution across a fieldof-view larger than the largest object to be imaged. We have sought to meet these requirements by designing a small ring diameter, depth-of-interaction (DOI) PET scanner for imaging animals up to $6 \mathrm{~cm}$ in diameter. "ATLAS" (Advanced Technology Laboratory Animal Scanner) is based on "phoswich" detector modules constructed from two optically connected, but different, "fast" scintillator layers coupled to miniature position-sensitive photomultiplier tubes

Jürgen Seidel is with the National Institutes of Health, Bethesda, MD, 20892, USA (telephone: 301-496-5675, e-mail: jurgen_seidel@nih.gov).

Juan José Vaquero was with the National Institutes of Health, Bethesda, MD, 20892, USA. He is now with the Hospital General Universitario Gregorio Marañón, 28007 Madrid, España (telephone: +34 91586 6678, email: juanjo@mce.hggm.es).

Michael V. Green is with the National Institutes of Health, Bethesda, MD, 20892, USA. (telephone: 301-496-5675, e-mail: mgreen@mail.cc. nih.gov).
(PSPMTs). The scintillator-of-interaction is identified by measuring the decay time (LGSO: $40 \mathrm{~ns}$, GSO: $60 \mathrm{~ns}$ ) of the light pulse of each event [1]. With this technique it is possible to have a substantial total crystal depth $(15 \mathrm{~mm})$ while at the same time having apparently short crystals ( 7 $\mathrm{mm}$ LGSO and $8 \mathrm{~mm}$ GSO) that minimize the DOI effect at small ring diameters. The small ring diameter, in turn, further improves sensitivity by increasing the system solid angle.

While the ATLAS design exploits well-known principles for minimizing the DOI effect and increasing sensitivity, the degree to which this strategy achieves these goals compared to simpler schemes is also important. The construction of phoswich modules requires additional fabrication steps and necessitates other electronic and data processing changes that complicate the design of this machine. These complications could be avoided if the same performance could be achieved by a scanner without DOI capability and with only a single crystal type. Accordingly, we utilized the Monte Carlo simulator created to study the characteristics of ATLAS to evaluate LSO scanners with identical aperture and axial FOV but having crystals of different depths and no DOI capability.

\section{MATERIALS AND METHODS}

ATLAS (Figure 1) consists of 18 LGSO/GSO (Hitachi) phoswich detector modules arranged around a ring $11.8 \mathrm{~cm}$ in diameter [2]. Each module is comprised of a $9 \times 9$ array of 2 $\mathrm{mm}$ square x $15 \mathrm{~mm}$ deep phoswich elements $(2.25 \mathrm{~mm}$ pitch), each of which is composed of a $7 \mathrm{~mm}$ long LGSO crystal optically glued end-on to an $8 \mathrm{~mm}$ long GSO crystal. The GSO end of the crystal bundle is optically glued to a miniature PSPMT (Hamamatsu R7600-C8).

The 18 detector modules are combined in groups of three to form a total of six sectors. The signals originating from the ATLAS sectors are fed into a data acquisition system supplied by A \& D Precision Co. (Newton, MA 02460, USA). The system consists of six charge integrating ADC modules [3] with custom modifications to facilitate identification of scintillation decay times, one scaler module, a custom coincidence logic controller, and a high-speed PCIbus interface card. The controller detects coincidences between sectors and initiates signal integration and ADC read out. The data are collected by two alternating $128 \mathrm{kB}$ memory buffers on the PCI-bus card in a dual-processor PC operating under the Linux OS. 
A Monte Carlo code was written to simulate the geometry and physical response of the ATLAS system to $511 \mathrm{keV}$ annihilation radiation. The simulation included modeling of the polygonal detector geometry as well as the effects of positron range (F-18), annihilation photon noncollinearity, and Compton scattering within scintillation crystals and between scintillator layers. Linear attenuation coefficients for LSO and GSO were derived as a function of photon energy from atomic photon cross section tables and the known atomic composition of the scintillators. For the purpose of this simulation, LGSO was regarded to have the same properties as LSO.

The $0.25 \mathrm{~mm}$ gaps between phoswich elements were not modeled. Instead, in order to simplify the simulation, an array was treated as a continuous block of $2.25 \mathrm{~mm}$ wide crystals each directly touching its neighbors. This strategy causes ACS to be overestimated and radial resolution to be slightly underestimated. Sensitivity values were corrected for this effect by reducing the simulated ACS values by the product of the geometric fill-factors of the real arrays. No corrections were applied to the radial resolution estimates since their primary purpose here was to allow comparison of systems rather than accurately determine their absolute spatial resolution.

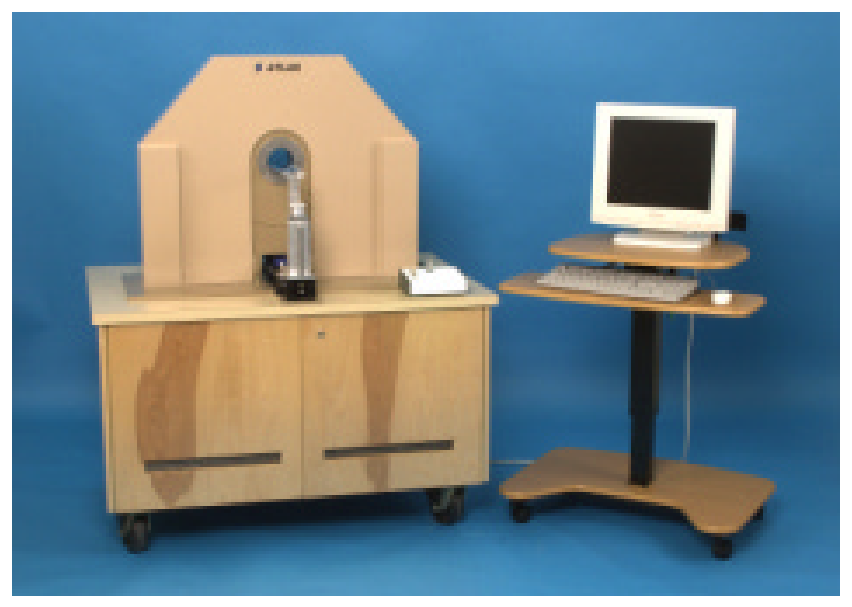

Figure 1. The ATLAS small animal PET scanner.

\section{A. Simulated Resolution Uniformity}

Coincidence data were acquired from a simulated point source of $511 \mathrm{keV}$ annihilation radiation moved in $1 \mathrm{~mm}$ increments from the geometric center of the system to $32 \mathrm{~mm}$ off the central axis. Events were considered valid if both absorbed photons deposited more than $250 \mathrm{keV}$ in their respective detectors. Coincidences occurring between LGSOLGSO crystals, LGSO-GSO crystals and GSO-GSO crystals were binned into separate sinograms. The polygonal ATLAS detector geometry exhibits small gaps between detector modules. As a consequence, small gaps are present in the sinograms which are compensated for by using the constrained Fourier space method [4]. These data were reconstructed into images using 2D filtered backprojection and a ramp filter.

The code was then modified to create three LSO scanners that differed from the ATLAS design only in the depths of their scintillation crystals: $15 \mathrm{~mm}, 10 \mathrm{~mm}$ and $7 \mathrm{~mm}$ of LSO. The same point source data obtained for ATLAS were then acquired for each of these simulated scanners. Note that in these scanners, no DOI information is available.

\section{B. Measured Resolution Uniformity}

The variation in resolution with radius for events depositing more than $250 \mathrm{keV}$ in each detector was measured for the actual ATLAS scanner using a $0.5 \mathrm{~mm}$ diameter Na22 point source at the center of a $25 \mathrm{~mm}$ diameter solid plastic ball. The source was imaged every two millimeters out to $31.3 \mathrm{~mm}$ starting $1.3 \mathrm{~mm}$ from the aperture center. Images of the source at each position were reconstructed in the same manner as in the simulation.

Radial and tangential spatial resolutions were calculated from the apparent width (FWHM) of the point sources in all data sets as a function of radial position. The width of the pixels in the reconstructed images was $0.28 \mathrm{~mm}$ and thus was considered small enough to have a negligible effect on the point source FWHM.

\section{Simulated Absolute Central Point Source Sensitivity}

The fraction of annihilations yielding detected coincidence events was determined for a point source located at the geometric center of each simulated scanner for energy thresholds of 100 and $250 \mathrm{keV}$.

\section{Measured Central Point Source Sensitivity}

A $1.2 \mathrm{~mm}$-diameter F-18 line source two centimeters long and surrounded by a $2 \mathrm{~mm}$ thick lucite annulus was placed co-linearly with the central axis of the ATLAS scanner exactly spanning the axial field-of-view. The fraction of annihilations yielding valid coincidences above $100 \mathrm{keV}$ and $250 \mathrm{keV}$ was determined and ACS calculated with the relation: ACS $(\%)=2 \times$ central line source sensitivity where central line source sensitivity is the coincidence counts/sec for the given energy condition/total microCuries in the $2 \mathrm{~cm}$ long line source $/ 37,000$ counts $/ \mathrm{sec} / \mu \mathrm{Ci}$.

\section{RESULTS}

Plots of radial resolution vs. radius measured for the real and for the simulated ATLAS scanner are compared to similar curves obtained for the three simulated LSO scanners in Figure 2. Absolute central point source sensitivity estimates are summarized in Table 1.

TABLE 1.

Absolute Central Point Source Sensitivity

\begin{tabular}{cccccc}
\hline $\begin{array}{c}\text { Threshold } \\
(\mathrm{keV})\end{array}$ & $\begin{array}{c}\text { LSO } \\
7 \mathrm{~mm}\end{array}$ & $\begin{array}{c}\text { LSO } \\
10 \mathrm{~mm}\end{array}$ & $\begin{array}{c}\text { LSO } \\
15 \mathrm{~mm}\end{array}$ & $\begin{array}{c}\text { ATLAS } 15 \mathrm{~mm} \\
\text { Phoswich } \\
\mathrm{S}^{*}\end{array}$ & $\mathrm{M}^{*}$ \\
\hline 100 & $1.3 \%$ & $2.1 \%$ & $3.2 \%$ & $2.8 \%$ & $2.7 \%$ \\
250 & $0.9 \%$ & $1.5 \%$ & $2.4 \%$ & $2.0 \%$ & $1.8 \%$ \\
\hline \multicolumn{5}{c}{$\mathrm{S}^{*}:$ simulated; $\mathrm{M}^{*}:$ measured. }
\end{tabular}




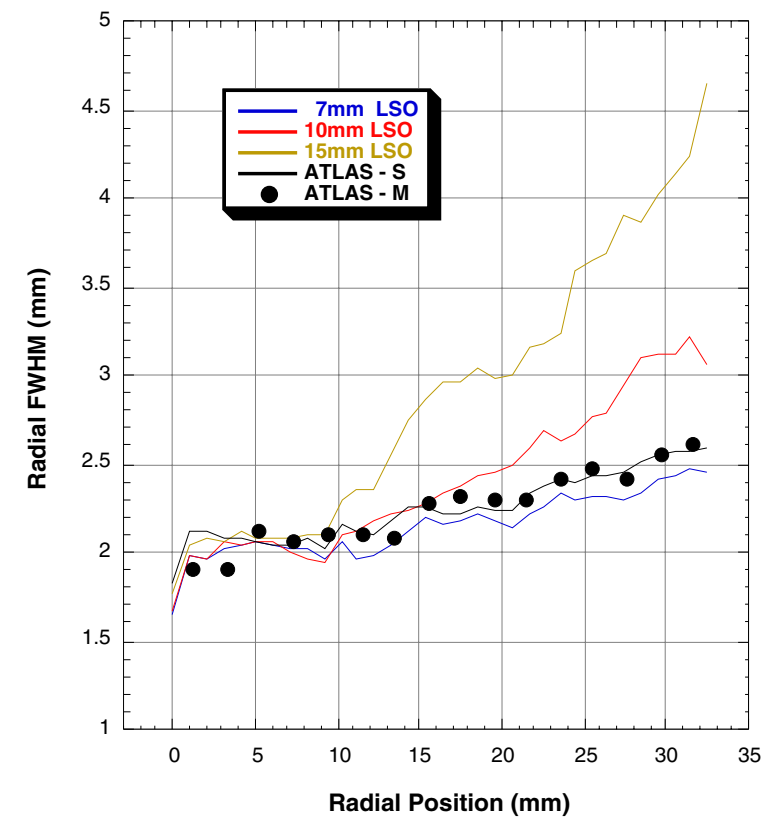

Figure 2. Radial resolution vs. radius for different scanners. ATLAS-S: simulated ATLAS results; ATLAS-M: measured ATLAS results.

Tangential resolution did not vary by more than $10 \%$ for any of the scanners over the central $6 \mathrm{~cm}$ FOV and these results are not shown. Forty-three percent of all coincidences were identified as LGSO-LGSO, 44\% as LGSO-GSO and $13 \%$ as GSO-GSO. These values were used as weighting factors to combine the three spatial resolution estimates obtained for the different coincidences into the single ATLAS radial resolution curve shown in Figure 2.

\section{Discussion}

The results portrayed in Figure 2 and Table 1 suggest that ATLAS achieves simultaneously good sensitivity and resolution uniformity compared to equivalent single scintillator designs without DOI capability. While the 15 $\mathrm{mm}$ LSO scanner possesses higher sensitivity, and the $7 \mathrm{~mm}$ scanner slightly better resolution uniformity, neither of these devices matches ATLAS's sensitivity and resolution uniformity at the same time. The $10 \mathrm{~mm}$ LSO scanner perhaps the best overall match to ATLAS - exhibits a $51 \%$ resolution variation $3 \mathrm{~cm}$ off-axis that is nearly a factor of two poorer than ATLAS (27\%) and is $25 \%$ less sensitive. MicroPET [5], an existing $17.2 \mathrm{~cm}$ diameter, $10 \mathrm{~mm}$ deep LSO scanner, exhibits the same spatial resolution at $3 \mathrm{~cm}$ as ATLAS, but with $0.6 \%$ sensitivity $(250-650 \mathrm{keV}$ energy window).

The simulated ATLAS radial resolution variation (curve $S$, Figure 2) and ACS (Table 1) are in close agreement with the measured resolution variation (M, Figure 2) and ACS (Table 1) of the actual scanner. These results illustrate the internal consistency of these data and support the validity of the simulation code.

Measured ACS for the full ATLAS ring (1.8\%) is slightly higher than our previous experimental estimate of ACS made on a partially completed ATLAS detector ring (1.6\%, [2]). This difference can be attributed to the escape of some positrons from the glass capillary tube $(0.2 \mathrm{~mm}$ wall thickness) used in the previous study. Annihilation of these positrons takes place mostly outside the scanner's field-ofview and they remain undetected by the scanner whereas such events are usually included in the dose calibrator measurement. Our simulation shows that the additional two $\mathrm{mm}$ thick lucite annulus surrounding the line source leads to $11 \%$ more detectable annihilations, almost exactly the difference between the past [2] and present ACS measurements.

The absolute magnitude of spatial resolution shown in Figure 2 and the apparent variation of resolution across the FOV depend, in part, on spatial sampling. In the present study simulated and measured values of resolution were obtained with the detector ring stationary so that spatial sampling is relatively poor and resolution is degraded, particularly in the central FOV. However, in the actual ATLAS scanner the detector array can be mechanically "wobbled" at rates up to 8 revolutions per second. Under such circumstances (which have yet to be fully evaluated) it is expected that central FOV resolution will be improved by as much as $20 \%$. Other strategies to enhance spatial sampling (and spatial resolution) by more fully exploiting the unique geometry of the phoswich design are also under investigation.

Additional advantages accrue from the ATLAS phoswich design: the number of lines of response is substantially greater than for non-DOI scanners [5] thereby improving offcenter spatial sampling; axial, as well as radial, resolution uniformity is also improved by phoswich DOI compensation, a useful feature if the ATLAS axial FOV is ever increased by adding additional detector rings; radial spatial resolution should, itself, be improved by allowing coincidences to be rejected that are comprised of intra-module scatter events that contain a mixture of light decay times or that are positioned between phoswich centers [6] (a feature not exploited in this study).

Finally, coincidence data acquired by ATLAS will be reconstructed routinely using $3 \mathrm{D}$ iterative, resolution recovery reconstruction algorithms [7] rather than $2 \mathrm{D}$ or $3 \mathrm{D}$ FBP. With such methods, it should be possible to capitalize on ATLAS's sensitivity and improve absolute spatial resolution substantially beyond that shown in Figure 2.

\section{CONCLUSIONS}

The present study supports the view that the ATLAS design achieves simultaneously good resolution, resolution uniformity and sensitivity over a $6 \mathrm{~cm}$ FOV compared to non-DOI LSO scanners of similar aperture and axial FOV.

\section{ACKNOWLEDGMENT}

The authors are deeply indebted to James Sullivan, Paul Fitze, Jimmie Powell, Franklin Sharpnack II, and Carroll Toms of the Mechanical Instrument Design \& Fabrication Section and to Burton Chidakel, Computer \& Electronics Section, Office of Research Services, NIH for their help with the ATLAS project. The authors also thank Raymond Ford, Division of Engineering Services, NIH, and Fernando J. Barbosa and William Gunning, Thomas Jefferson National Accelerator Facility.

We gratefully acknowledge the assistance of Val Zavarzin, A \& D Precision Co., with customizing the data acquisition 
system to our needs. We thank Gerd Muehllehner for drawing our attention to the effect of positron escape on the measurement of ACS.

\section{REFERENCES}

[1] J. Seidel, J. J. Vaquero, S. Siegel, W. R. Gandler, and M. V. Green, "Depth Identification Accuracy of a Three-Layer Phoswich PET Detector Module", IEEE Trans Nucl Sci, vol. 46, no. 3, pp. 485-490, 1999.

[2] J. Seidel, J. J. Vaquero, I. J. Lee, and M. V. Green, "Experimental Estimates of the Absolute Sensitivity of a Small Animal PET Scanner with Depth-of-Interaction Capability," in Conference Record of the 2000 IEEE Medical Imaging Conference, paper M21-57.

[3] V. G. Zavarzin and W. A. Earle, "A 500k Event/s 12-Bit ADC System with high-speed buffered PCI Interface," in Conference Record of the 1998 IEEE Nuclear Science Symposium, paper N20-25.

[4] J. S. Karp, G. Muehllehner, D. A. Mankoff, C. E. Ordonez, J. M. Ollinger, M. E. Daube-Witherspoon, A. T. Haigh, D. J. Beerbohm, "Continuous-Slice PENN-PET: A Positron Tomograph with Volume Imaging Capability", J Nuc Med, vol 31, no. 5, pp. 617-627, 1990

[5] A. F. Chatziioannou, S. R. Cherry, Y. Shao, R. W. Silverman, K. Meadors, T. H. Farquhar, M. Pedarsani, and M. E. Phelps, "Performance Evaluation of MicroPET: a High-resolution Lutetium Oxyorthosilicate PET Scanner for Animal Imaging", J Nuc Med, vol. 40, no. 7, pp. 1164-1175, 1999.

[6] J.J. Vaquero, J. Seidel, S. Siegel, W.R. Gandler and M.V. Green, "Performance Characteristics of a Compact Position-Sensitive LSO Detector Module", IEEE Trans Med Imag, vol. 17, no. 6, pp.967-978, 1998.

[7] C. A. Johnson, J. Seidel, R. E. Carson, W. R. Gandler, A. Sofer, M. V. Green, and M. E. Daube-Witherspoon, "Evaluation of 3D Reconstruction Algorithms for a Small Animal PET Camera", IEEE Trans Nucl Sci, vol. 44, no. 3, pp. 1303-1308, 1997. 